\title{
Contact, nanoindentation, and sliding friction
}

\author{
A. Buldum and S. Ciraci \\ Department of Physics, Bilkent University, Bilkent 06533, Ankara, Turkey \\ Inder P. Batra \\ IBM Almaden Research Center, San Jose, California 95120-6099
}

(Received 3 June 1997)

\begin{abstract}
This paper presents an atomic-scale study of contact, indentation, and subsequent pulling and dry sliding of a sharp and blunt metal tip on a metal surface. The evolution of atomic structure and the variation of perpendicular and lateral forces are calculated by molecular-dynamics methods using an empirical potential based on the embedded-atom model. The sharp tip experiences multiple jumps to contact in the attractive force range. The contact interface grows discontinuously mainly due to disorder-order transformation leading to disappearance of a layer and hence abrupt changes in the normal-force variation. Atom exchange occurs in the repulsive range. During the pulling off, the connective neck is reduced discontinuously; however, not all the abrupt changes of the pulling force are associated with the creation of a new layer in the neck. The sliding of the sharp tip (or single asperity) induces two consecutive structural transformations that occur periodically, but end with the wear of a layer. The situation for a blunt tip is, however, quite different. [S0163-1829(98)02203-6]
\end{abstract}

\section{INTRODUCTION}

Scanning tunneling microscopy (STM) and atomic force microscopy (AFM) have great impact on the development of nanoscience. In particular, the atomic scale investigation provided by these new techniques have been the source of motivation for the recent studies of point contact and nanoindentation, as well as the sliding friction. The relevant length scales in the contact or in the connective neck between the tip and the sample can be in the range of the Fermi wavelength $\lambda_{F}$, whereby the quantum size effects become pronounced. Moreover, the discrete structure made from individual atoms dominates over the continuum description in this length scale. Any change in the atomic structure of a point contact can lead to significant changes in its mechanical and electrical properties. The level spacings of transversally confined electrons can be as large as $\sim 1 \mathrm{eV}$. Therefore, their quantization are expected to lead to discrete variations of various observables (such as conductance, surface energy, elastic stiffness, Young's modulus, etc.) with the change of the confining size. ${ }^{1}$ Perhaps, even the change of diameter during deformation is affected with the quantization. ${ }^{1}$ As a matter of fact, the two-terminal conductance $G$ has shown discrete variations as the contact grows by pushing ${ }^{2}$ (or as the connective neck reduces by pulling off ${ }^{3}$ ). Recently, the simultaneous measurement ${ }^{4,5}$ of the conductance and force variations with the stretch of the connective neck have established that observed features are closely related to the discrete variation of the cross section $A$ at the contact, as in fact proposed by the earlier theoretical studies. ${ }^{6,7}$ The detailed analysis of the contact formation and the atomic rearrangements are important not only for understanding the contact resistance $(R=1 / G)$, but also for contact mechanics, ${ }^{8}$ wear and tip-sample interaction effects in AFM. ${ }^{9}$

The dry sliding friction between two metal surfaces, that are in direct contact through their asperities involve also many complex phenomena. ${ }^{10,11}$ While the relative motion of two commensurate surfaces can take place through the repeating stick-slip motion, ${ }^{12}$ the situation becomes complicated if the sliding surfaces are incommensurate. ${ }^{13,14}$ It has been argued that frictionless sliding (or superlubricity) can occur if the surfaces are flat and incommensurate. ${ }^{15}$ On the other hand, the study of sliding friction between the Ir tip and the $\mathrm{Au}(111)$ surface did not reveal atomic scale stick-slip behavior, but found stronger friction during unloading then during loading with the identical values of the loading force. ${ }^{16}$ The formation of the contact between an asperity and the substrate surface, and its evolution by the wear occurring in the sliding motion involve physical events that are common to the nanoindentation. ${ }^{17-19}$

Earlier the tip-sample interaction effects have been studied to reveal the modification of electronic structure at the close proximity of the tip. ${ }^{20}$ The variation of the electronic potential, interaction energy, perpendicular and lateral force variations were calculated as a function of the tip-sample distance by using $a b$ initio, self-consistent field pseudopotential method within the local-density approximation., $9,18,20-23$ In these studies not only a clear picture of the collapse of barrier and transition from tunneling to ballistic transport have been developed, but also the nature of short-range and long-range forces and their variations with the distance have been clarified. Furthermore, the two-terminal conductance through a junction between a metal tip and sample were calculated as a function of the contact area $A$; the effect of material parameters were discussed. ${ }^{6,7,22}$ The classical molecular-dynamics (CMD) method with empirical potential were used to calculate the images of force in AFM, ${ }^{24}$ to investigate atomic scale modifications at the close proximity of the tip and to simulate the formation of a connective neck. ${ }^{7,17,25-27}$ In spite of the relatively shorter time scale in the simulation studies based on the CMD method, several important aspects of the contact were established.

The emphasis of the present work is focused on the evolution of the atomic structure in the course of contact, inden- 


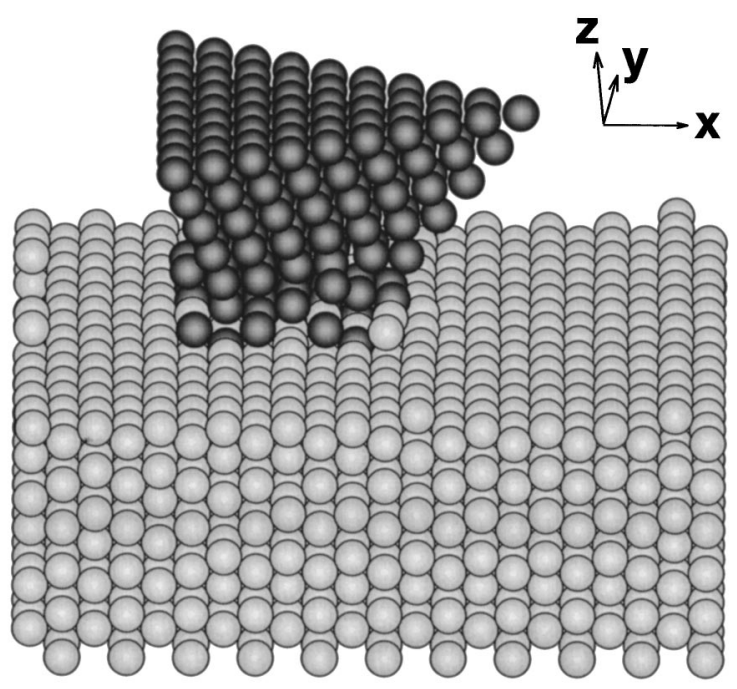

FIG. 1. Schematic description of the Ni(111) tip (or asperity) and the $\mathrm{Cu}(110)$ substrate. The dark and light gray balls represent the $\mathrm{Ni}$ and $\mathrm{Cu}$ atoms, respectively. The $\mathrm{Ni}(111)$ and $\mathrm{Cu}(110)$ atomic planes are parallel to the $x y$ plane. The long side of the (110) rectangle is parallel to the $x$ axis.

tation, pulling off and sliding, and its effects on the normal $\left(F_{N}\right)$ and lateral $\left(F_{L}\right)$ force variations. In particular, we examine the effects of various parameters (such as the shape of the tip, and its structure) on the formation of the contact and reveal new mechanisms in the junction growth. The first part of the paper deals with the contact and indentation, subsequent pulling off and sliding of a sharp $\mathrm{Ni}(111)$ tip on the relatively soft $\mathrm{Cu}(110)$ substrate. In the second part, we study the indentation and sliding of a blunt $\mathrm{Ni}(001)$ tip on the $\mathrm{Cu}(001)$ surface. The tip considered here is relevant for AFM, as well as for the asperity in dry sliding friction. We use a state of the art CMD method and embedded atom potential $^{28}$ and provide a detailed analysis about how the atomic rearrangements occur and which structural features are retained under excessive strain.

\section{SHARP TIP}

The sharp tip (or asperity) is formed from ten $\mathrm{Ni}(111)$ layers that has a single atom at the apex. The substrate has fourteen $\mathrm{Cu}(110)$ atomic planes which are parallel, but incommensurate to the $\mathrm{Ni}(111)$ planes. The atomic arrangement of the tip-sample system after the contact is shown in Fig. 1. The top two layers of the tip are robust; either they are lowered or raised in increments of $\Delta z=0.1 \AA$ along the $z$ direction by keeping the $(x, y)$ positions of the atoms also fixed, or they are translated in increments of $\Delta x=0.05 \AA$ along the $x$ axis in the sliding. The bottom four layers of the substrate are fixed. Between two consecutive increments $(\Delta x$ or $\Delta z$ ) all the dynamic atoms of the asperity (120 atoms) and the substrate (1650 atoms) are relaxed to attain their steadystate positions for 3000 steps in pulling off and in pushing (and for 500 steps in sliding). In each time step, $(\Delta t$ $=10^{-15}$ seconds) the positions and the velocities of all dynamic atoms that move under the force generated from the interatomic potential are determined by solving Newton's equations of motion. The temperature is kept fixed at $4 \mathrm{~K}$ by

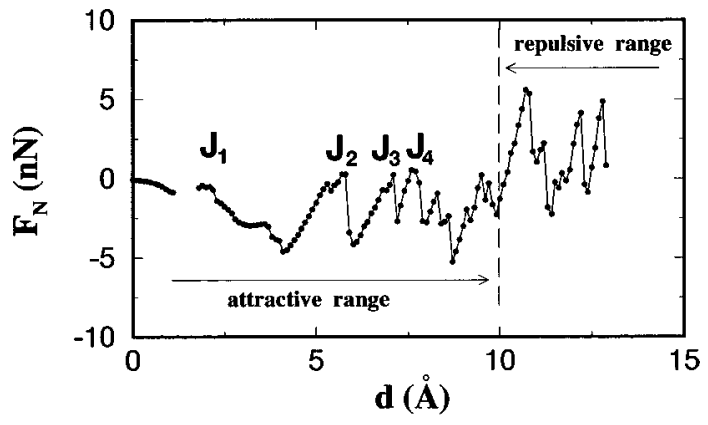

FIG. 2. Variation of the normal force $F_{N}$ versus the downward displacement (or push) $d . J_{1}, J_{2}, \ldots$ indicate the features associated with sudden change in $F_{N}$. The attractive and repulsive range of $F_{N}$ are indicated.

rescaling the velocities of all dynamic atoms at the end of each step. To ensure that the above systems are converged to steady state after each increments $(\Delta x$ or $\Delta z)$ we calculated the physical quantities, such as temperature, force, kinetic and potential energy in each relaxation step and traced their variation. We observed no significant variation of the above quantities except their natural fluctuations before the preset maximum number of relaxation is reached.

\section{A. Contact and indentation}

The sharp tip is pushed when its separation from the $\mathrm{Cu}(110)$ surface is $4 \AA$. In Fig. 2 we show the variation of the normal force $F_{N}$ (along the $z$ direction) with the push (or downwards displacement of the tip, $d=p_{d} \Delta z, p_{d}$ being the number of increments). The sign of the attractive force is taken negative. The atomic structure of the junction are illustrated in Fig. 3 for relevant stages of the contact and indentation. At large separation, $F_{N} \sim 0$ owing to the shortrange nature of interaction forces; but it becomes attractive and increases with increasing $d$, passes through a minimum and then decreases. The interaction of the single $\mathrm{Ni}$ atom at the apex, that faces the substrate, dominates the force variation. In agreement with the $a b$ initio calculations ${ }^{21} F_{N}$ decreases because the apex of the Ni tip enters the repulsive range. The electronic contact, where the electronic structure and hence electronic charge density are modified owing to the interaction between outermost $\mathrm{Ni}$ and $\mathrm{Cu}$ atoms, sets in for $d>1.3 \AA$. For $d \gtrsim 3 \AA$, the effective potential barrier $\Phi_{\text {eff }}$ collapses to allow the ballistic conductance. The first fall of $F_{N}$ denoted by $J_{1}$ corresponds to the jump of the apex $\mathrm{Ni}$ from its metastable position to the hollow $(H)$ site. This situation indicates that a mechanical contact is established between the tip and the sample surface. At $d=4.3 \AA$ four $\mathrm{Ni}$ atoms (apex atom and three atoms in the second layer of the bare asperity) move to the $H$ sites of the substrate and become in registry with the $\mathrm{Cu}(110)$ planes. The $\mathrm{Ni}$ atoms in the layer following the contact occupy bridge sites and keep their hexagonal structure, but their registry with the adjacent $\mathrm{Ni}$ layers of the asperity is destroyed. The attractive force decreases quasilinearly with the increasing push in the region $4.3 \AA<d<5.7 \AA$. At $d \sim 5.8 \AA$ the atomic structure and the layers near the contact becomes disordered and eventually one layer of the Ni asperity disappears. This is a yield that causes to the sudden fall of $F_{N}$ labeled by $J_{2}$. 


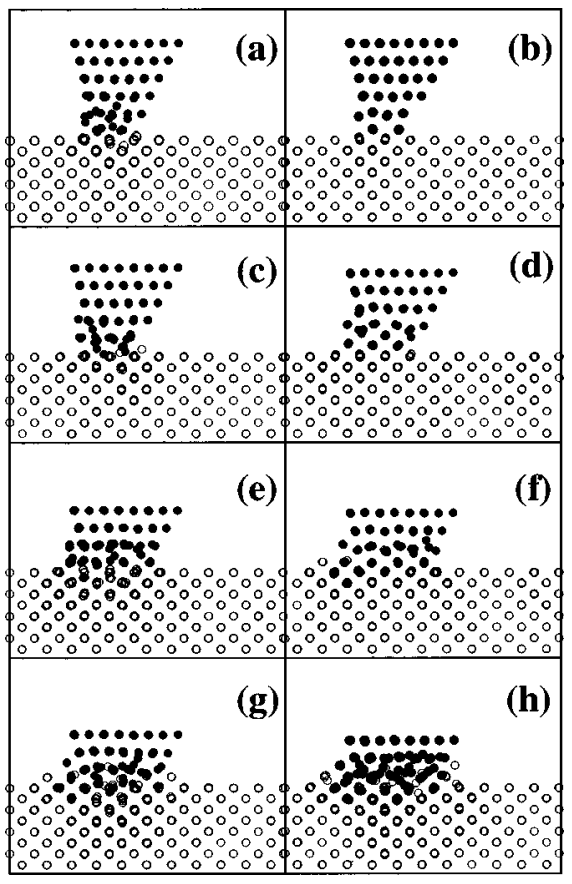

FIG. 3. Side views of the atomic structure for the Ni(111) tip (or asperity) and the $\mathrm{Cu}(110)$ substrate are illustrated for various stages of the indentation. The tip is pushed from a distant separation towards the sample surface in increments of $\Delta z$. The downwards displacement of the tip is $d=p_{d} \Delta z$. (a), (b), (c), (d), (e), (f), (g), and (h) correspond to $d=7.1,7.2,8.0,8.7,11.1,11.5,12.2$, and $12.9 \AA$, respectively. The $\mathrm{Ni}$ and $\mathrm{Cu}$ atoms are shown by filled and empty circles, respectively.

Between $J_{2}$ and $J_{3}, F_{N}$ decreases again with $d$ (or push), and displays also a quasilinear variation; the $\mathrm{Cu}$ atoms at the contact are gradually displaced from their original positions, and the atoms in the first layer lose their registry. This situation lasts until $d=7.2 \AA$, where the area of the contact increases and the first $\mathrm{Ni}$ layer becomes in registry with the substrate. At the end, the attractive $F_{N}$ increases suddenly. Further variation of $F_{N}$ is not regular (for $d>7.2 \AA$ ), and attractive $F_{N}$ generally decreases with increasing $d$ (or $p_{d}$ ) if the number of layers does not change. On the other hand, the attractive $F_{N}$ increases if the two adjacent layers of the asperity merges into one layer, or the number of asperity atoms at the contact increases or disordered atomic structure becomes ordered. It is seen that for $0<d<10 \AA, F_{N}(d)$ is generally in the attractive range; displays multiple (or sequential) jump-to-contact phenomena in the attractive range. Tip atoms having relatively lower coordination can lower the total energy by moving to the substrate surface and forming a commensurate layer. The growth of the contact is easier for a sharp tip as in the present case. Moreover, while the repulsive forces act on the contact layer, the atoms in the layer above the contact become in the attractive range. Initially, the contact area $A$ is relatively smaller; the resulting force becomes attractive. Beyond certain value of $A$ (i.e., contact involving $23 \mathrm{Ni}$ and $\mathrm{Cu}$ atoms and thus $d>10 \AA) F_{N}(d)$ varies but always stays in the repulsive range. The elastic stiffness constants of the bulk Ni along [111] direction and that of the bulk $\mathrm{Cu}$ along [100] direction are $\left(C_{11}+2 C_{12}\right.$ $\left.+4 C_{44}\right) / 3=3.6 n N / \AA^{2}$ and $C_{11}=1.7 n N / \AA^{2}$, respectively. Both values are relatively larger than that of the junction (a)

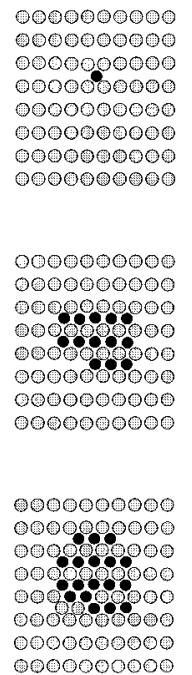

(b)

๑00000000 10000000000 0000900000 000090000 0000000000 0000000 00000000

(c)

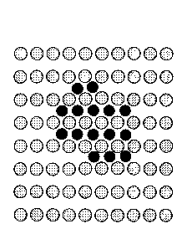

(d)

(e)

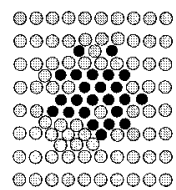

(f)

FIG. 4. Top views of the atomic structure at the interface of the contact are shown for various stages of contact and indentation. Substrate atoms in the surface and tip atoms in the contact interface are shown by large gray and small dark circles, respectively. The panels (a), (b), (c), (e), and (f) correspond to $d=0.9,5.2,8.7,9.2$, 9.9 , and $11.5 \AA$, respectively. The atom exchange are seen in the panels in (e) and (f).

estimated from Fig. 2. This implies that the discrete nature of the junction and its atomic structure becomes crucial for the small contacts.

The atom exchange, ${ }^{19}$ in which substrate atoms $(\mathrm{Cu})$ raise to the contact interface (i.e., first layer above the substrate surface) and to the layers above it while the $\mathrm{Ni}$ atoms of the asperity dip into the substrate, starts to occur at the beginning of the repulsive range and increases with the deformation of the substrate. This process contribute to the growth of $A$. The contact area grows discontinuously; in different stages of the contact and subsequent indentation the contact interface incorporates $1,4,8,13,15,18,23, \ldots$ atoms. Interestingly, the growth of the contact in terms of new atoms brought into the interface, i.e., $A_{i+1}-A_{i}$, does not present a regular pattern and involves more then one atom. The dissapearance of an asperity layer gives rise to a sudden change of the force, i.e., sudden increase (decrease) in the attractive (repulsive) range. However, not all the sudden changes of $F_{N}(d)$ are associated with the dissapearance of a layer. The evolution of the contact is illustrated by Fig. 4, where we note that the significant deformation of the interface starts to occur in the repulsive force range.

The simulation of indentation ${ }^{25}$ in the $\mathrm{Au}(001)$ [also $\mathrm{Ni}(001)$ ] sample produced by the blunt $\mathrm{Ni}(001)$ [also $\mathrm{Au}(001)]$ tip at $T=300^{\circ} \mathrm{K}$ revealed that the deformation occurs in the softer metal. In the present study the sequential (multiple) jump-to-contact phenomena and the deformation induced therefrom occur in the sharp tip even it is harder than the sample. As seen in Fig. 2, the multiple jump-tocontact phenomena yield an average attractive force in a rather long-range displacement of the sharp tip even though the deformed apex of the tip is under the action of the repulsive force. The puzzling results ${ }^{29}$ of the measurements of force between the $\mathrm{Si}(111)$ tip and the $\mathrm{Si}(111)$ sample can be explained by the deformation of the sharp tip yielding the 


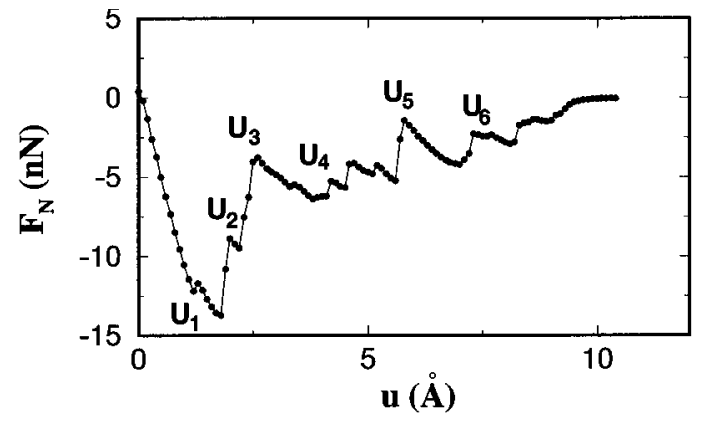

FIG. 5. Variation of the tensile force $F_{N}$ versus the upwards displacement or pull $u\left(u=p_{u} \Delta z\right)$ in a connective neck formed by the pulling-off the sharp tip from an indentation. $U_{1}, U_{2}, \ldots U_{6}$ are relevant features that are explained in the text.

force variation similar to that in Fig. 2. In order to examine the variation of two-terminal conductance through a point contact created by $\operatorname{Ir}(111)$ tip, Todorov and Sutton ${ }^{7}$ carried out atomic scale simulations of the indentation and found that the contact grows discontinuously. Present work provides a detailed analysis for the growth of the contact and its atomic structure.

\section{B. Pulling off}

At certain stage of the indentation we stop lowering the tip, and start to move it away from the substrate in the positive $z$ direction. This way the junction formed by indentation is stretched and a connective neck is formed. ${ }^{17,25,30}$ This neck becomes narrower with the stretch (or pull $u=p_{u} \Delta z, p_{u}$ being the number of increments) and eventually is broken. We investigated the pulling off the junction from two different stages of the indentation, described in Sec. II A, i.e., $d$ $=10.1 \AA$ and $d=13.6 \AA$. In the latter, $A$ is relatively larger and the deformation at the junction is more pronounced. We first consider the former case, i.e., small size indentation made by a sharp tip. In Fig. 5 we show the variation of the tensile force $F_{N}(u)$ with the stretching. Figures 6 and 7 show side and top views of the atomic structure at the relevant stages of the stretch and reveal new and interesting features.

In agreement with the earlier studies ${ }^{5-7,31} F_{N}(u)$ curve shows abrupt changes. However, in contrast to the earlier studies each of these changes are not associated with the creation of a new layer. Furthermore, these changes do not exhibit a regular pattern with $u$. On the other hand, the completion of a new layer give rise to an abrupt decrease in $F_{N}(u)$. Before the formation of a new layer the structure of the neck becomes disordered, and eventually a new layer is formed. This is the yielding of the neck. As pointed out by earlier studies, ${ }^{7,25,31,32}$ the yielding and subsequent creation of a new layer is mediated by the disorder-order transformations in the nanowires with cross section incorporating only a few atoms as in the present case. Between two consecutive yielding of the neck $F_{N}(u)$ increases linearly. That is attributed to the quasielastic straining of the neck. In the present case, where $T=4 \mathrm{~K}$ and the initial junction is narrow (or $A$ is small), disorder-order transformations are usually sudden, but require significant stretching; in the meantime, different intermediate situations (such as transformation of an incomplete layer or generation of interstitial atoms, etc.) occur. At

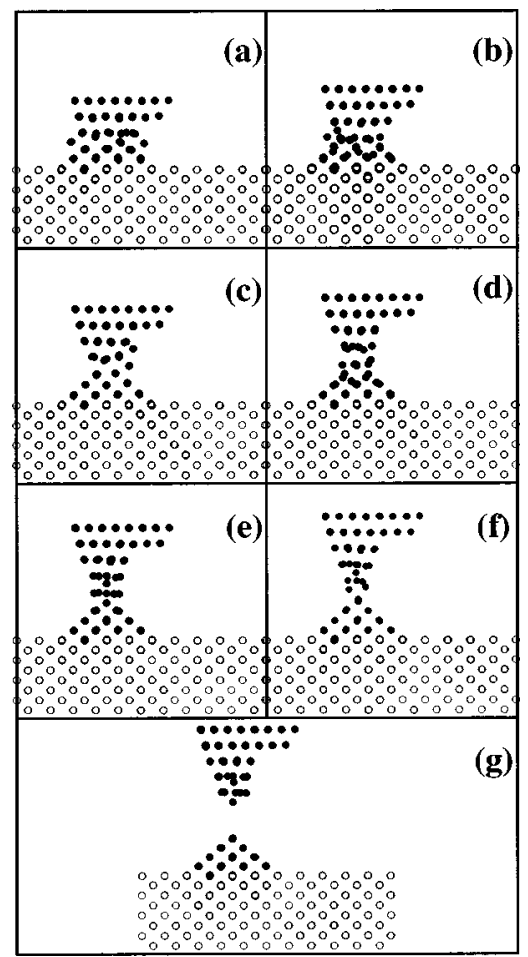

FIG. 6. Side-view snapshots of the atomic structure for the relevant steps of pulling the Ni tip from an indentation. The pullingoff starts from an indentation corresponding to $d=10.1 \AA$. The panels (a), (b), (c), (d), (e), (f), and (g) correspond to $u=0.7,2.1$, $4.2,5.6,6.3,7.7$, and $10.5 \AA$, respectively.

the beginning of the pulling of the junction, five dynamic layers of mainly $\mathrm{Ni}$ with some mixture of $\mathrm{Cu}$ atoms can be identified. For small $u, F_{N}(u)$ varies linearly; $\partial F_{N} / \partial u$ is large and hence requires a strong force to induce the first structural change $U_{1}$. However, $U_{1}$ displays an intermediate situation, and only after $U_{2}$ an additional layer is identified. Between $U_{1}$ and $U_{2}, \partial F_{N} / \partial u$ is reduced, and continues to reduce in the following quasielastic stages. The next new layer forms at $U_{3}$ and stays stable until $U_{4}$, but transition from seven-layer to eight-layer structure is completed between $U_{4}$ and $U_{5}$. After $U_{5}$, the junction has eight Ni layers above the substrate. Interestingly, the neck is formed from two pentagons (layers 4 and 5) and a quadrangle (sixth layer). Single atoms located between these layers form a string passing through the centers of pentagons and quadrangle. This low-temperature intermediate structure (a)

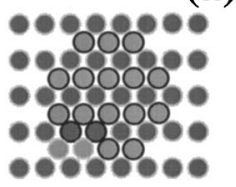

(b)

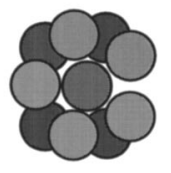

(c)

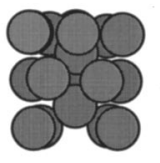

FIG. 7. Top and side views of the atomic structure during the formation of the connective neck. (a) Atomic structure of the substrate surface and the contact interface. Circles with the sharp and dotted circumferences are $\mathrm{Ni}$ and $\mathrm{Cu}$ atoms, respectively. In the gray scale, the higher the atoms are, the lighter become their color. (b) The top view of atoms at the neck forming pentagon, quadrangle and single atoms between them. (c) Side view of the neck. 
that seems to form at high strain and small cross section ${ }^{31,32}$ has been revealed by the present study for the tip retracting from an indentation. Implementation of a single atom between layers induces additional small structures in the force variation as if the mixture of elastic and yielding stages. As in the contact formation described in Sec. II A, the variation of $F_{N}(u)$ shortly before the break $(u>7 \AA$ in Fig. 5) is similar to that calculated for a single atom contact by using SCF pseudopotential method. ${ }^{18}$ The strongest attractive force in this range is $\sim 1.5 \mathrm{nN}$ that is also in agreement with our earlier ab initio calculations for single atom contact. ${ }^{18}$ In the course of stretch of the neck the structure of the interface (that is in contact with the substrate surface) does not change significantly and stays in registry with the $\mathrm{Cu}(110)$ structure. Moreover, the layers of the Ni pyramid that stayed on the substrate after the break, becomes also pseudomorphically commensurate with the $\mathrm{Cu}(110)$ structure. As for the Ni asperity that remains after the break of the neck, a few layers keep their hexagonal structure commensurate with the $\mathrm{Ni}(111)$ plane, except the pentagonal structure maintained at the bottom of the asperity.

Pulling from a higher level of indentation $(d=13.6 \AA$ in Sec. II A) starts from a junction that has relatively larger $A$ and more pronounced deformation, and displays similar features with following differences. In the latter the tensile force at the initial stage of stretch reach to higher level $\left(F_{N} \sim 14\right.$ $\mathrm{nN}$ ) owing to relatively larger contact area, but the amount of stretching achieved until the break is relatively smaller. In contrast to the previous pulling off from the low level of indentation (i.e., $d=10.1 \AA$ ), the Ni pyramid stayed on the substrate is relatively large while the $\mathrm{Ni}$ asperity becomes relatively smaller.

\section{Sliding friction}

To investigate the friction and wear of a single metal asperity on an incommensurate metal surface we used the same system that was considered in Secs. II A and II B. The asperity is first pushed to the $\mathrm{Cu}(110)$ surface along the $z$ direction and then is slit along the $x$ direction under the constant loading force $F_{N}=2.64 \mathrm{nN}$ distributed on the top plane of the asperity. The displacement of the asperity after $p_{s}$ increments is $s=p_{s} \Delta x$. The temperature is kept fixed at $4 \mathrm{~K}$.

Upon the formation of steady state contact between the $\mathrm{Ni}$ asperity and the $\mathrm{Cu}$ surface the energy of the whole system is lowered by $\sim 22 \mathrm{eV}$. The energy lowering originates mainly from the adhesive interaction. The atomic rearrangements during the development of the junction between the Ni asperity and substrate prior to sliding follows the similar events described in Sec. II A. The aspects that are relevant for the sliding are briefly summarized. Once the contact is established, the layer structure of the pyramid remains, but single atom at the apex and the subsequent layer disappears leaving only eight Ni layers including the contact layer (interface) at the surface of the substrate (as described in Fig. 1). While some $\mathrm{Ni}$ atoms substitute $\mathrm{Cu}$ atoms at the contact, some of them are accumulated behind the asperity. On the other hand, some $\mathrm{Cu}$ atoms migrate into the $\mathrm{Ni}$ asperity. The $\mathrm{Cu}(110)$ surface at the contact region can be viewed as a $\mathrm{Cu}-\mathrm{Ni}$ alloy with the lattice structure of the $\mathrm{Cu}(110)$ surface. In spite of the fact that the cohesive energy of $\mathrm{Ni} \sim 30 \%$ higher than

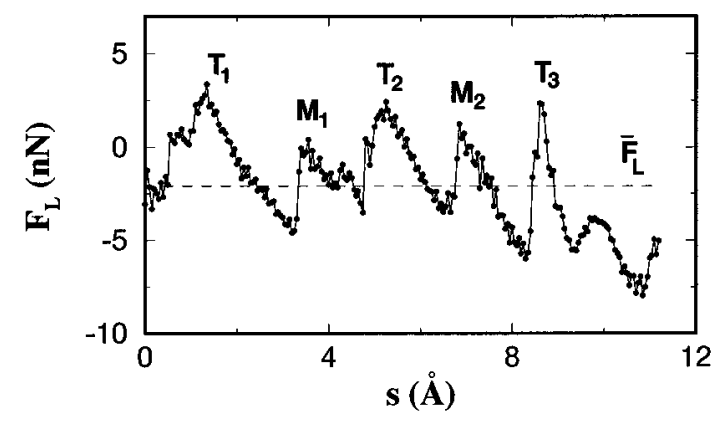

FIG. 8. Variation of the lateral force with the sliding $s$ of the $\mathrm{Ni}(111)$ asperity on the $\mathrm{Cu}(110)$ surface. The average lateral force $\bar{F}_{L}$ (or friction force) is shown by dashed line. $T_{n}$ and $M_{n}$ indicates different type structural transformation in the $n$th period. $F_{L}(s)$ opposing to the motion of the asperity is taken negative.

that of $\mathrm{Cu}$ crystal, two layers of the $\mathrm{Ni}$ pyramid which are adjacent to the $\mathrm{Cu}(110)$ surface have changed from the $\mathrm{Ni}(111)$ lattice structure and become matched the $\mathrm{Cu}(110)$ substrate. The distance between these layers comply with that of the $\mathrm{Cu}(110)$ planes. This is perhaps due to the lower coordination numbers of the $\mathrm{Ni}$ atoms near the apex. The remaining top five layers of $\mathrm{Ni}$ asperity keep their original $\mathrm{Ni}(111)$ lattice structure.

The sliding starts after the atomic configuration of the contact reached the steady-state condition; the asperity is translated by the increments of $\Delta x$. The variation of the lateral force $F_{L}$ with the displacement $s$ is shown in Fig. 8. By definition, the sign of $F_{L}(s)$ resisting to the sliding is taken negative. We note the following interesting features: (i) The abrupt changes of the $F_{L}(s)$ curve display a quasiperiodic variation with a period of approximately $3.5 \AA$. Two features of different characters (labeled $T$ and $M$ ) occur consecutively in each period; these are noticed by the fact that $F_{L}(s)$ decreases and changes sign with the displacement of the asperity. (ii) Between two consecutive features (from $T_{n}$ to $M_{n}$ or from $M_{n}$ to $\left.T_{n+1}\right) F_{L}(s)$ first decreases (where $F_{L}>0$ ) and then increases (where $F_{L}<0$ ) with displacement $s$; it displays a quasielastic behavior. (iii) The work done against $F_{L}(s)$ over a period from $T_{n}$ to $T_{n+1}$, i.e., $-\int_{T_{n}}^{T_{n+1}} F_{L}(s) d s$, increases from period to period. This deviation from the Amonton's law is explained by the growth of the junction. The variation of lateral force showing the elastic (or linear) region followed by an abrupt decrease is a peculiarity of the stick-slip process in dry sliding friction that occurred in certain ideal conditions so far. It is of particular interest since the sliding of a metal asperity on an incommensurate metal surface is shown to give rise to a stick-slip behavior. Since the asperity forms a finite-size contact with ordered structure, the occurance of a quasiperiodic stick-slip behavior cannot be explained by the sharpness of the asperity. It is rather due to the fact that the cubic lattice parameters of $\mathrm{Ni}$ and $\mathrm{Cu}$ vary within $\sim 2.5 \%\left(a_{\mathrm{Ni}}=3.52 \AA\right.$ and $\left.a_{\mathrm{Cu}}=3.61 \AA\right)$. Moreover all edges of the (111) unit cell has the same length as the shorter edge of the (110) unit cell $(a / \sqrt{2})$. Under these circumstances, the sliding of a $\mathrm{Ni}$ asperity follows a special path on the Born-Oppenheimer surface that passes through certain structural phases explained below. However, we expect that the stick-slip behavior in Fig. 8 can be washed out if the 
(a)

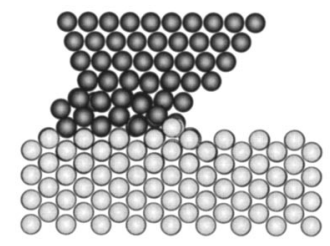

(c)

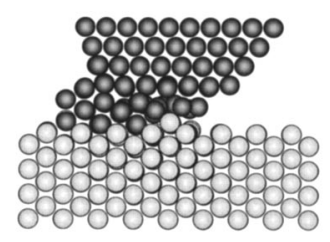

(e)

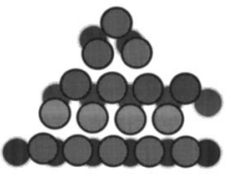

(f)

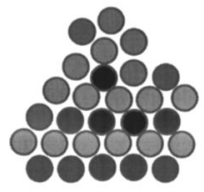

$(\mathrm{g})$

FIG. 9. Side view of atomic structure calculated for the Ni(111) asperity (dark gray circles) sliding on the $\mathrm{Cu}(110)$ surface (light gray circles). Panels (a) and (b) are before and after the structural transformation $T_{1}$; Panel (c) and (d) are before and after the structural transformation $M_{1}$. Only part of the substrate is shown. Panel (e) shows the $x y$ projection of the atoms in the Ni layer experiencing a structural transformation. Light gray circles correspond to the fifth $\mathrm{Ni}(111)$ layer from the top before $T_{1}$; after $T_{1}$ this layer is changed to the $\mathrm{Ni}(110)$ that is commensurate to the $\mathrm{Cu}(110)$ as shown by the dark circles with dotted circumference. Panel (f) shows the two $\mathrm{Ni}(110)$ layers corresponding to the situation in (c) before the transformation $M_{1}$. These two layers merged into one $\mathrm{Ni}(111)$ layer in panel $(\mathrm{g})$ as described by the side view (d) after $M_{1}$.

sliding involves multiple asperity. Very large contacts (that is unusual for an asperity) can give rise to misfit dislocation.

Snapshots of side views and $x y$ projections of the atoms in the layers where the sliding takes place are shown in Fig. 9, only for $T_{1}$ and $M_{1}$ features. Figure 9(a) depicts atomic configuration essentially before $T_{1}$, but after the onset of contact. As a result of sliding, the top four planes are displaced along the $x$ direction and also slightly lowered. This changed the registry between the fourth and fifth $\mathrm{Ni}(111)$ layers from the top; the energy of the system is raised and perhaps exceeded that of another structure. Eventually, as shown in Fig. 9(b), the fifth layer from the top, which was initially commensurate with the $\mathrm{Ni}(111)$ layers, undergoes a structural transformation and becomes matched the lower $\mathrm{Ni}$ layer that was already changed to the lattice structure of the $\mathrm{Cu}(110)$ plane. The $x y$ projection of atoms in this particular layer that changed its lattice structure through $T_{1}$ is shown in Fig. 9(e) before and after the transformation. One row of $\mathrm{Ni}$ atoms moved sideways, the next went ahead and third one merged to the lower layers. During this incommensurate- commensurate structural transformation, while part of the stored energy is kept in the new phase, the rest of it is released in the nonadiabatic transition and dissipated perhaps by exciting nonequilibrium phonons. The amount of energy dissipated in this transition is calculated $\sim 3 \mathrm{eV}$. The force $F_{L}(s)$, which was initially resisting the sliding, is lowered suddenly during the transition and changed its direction. The above variation of $F_{L}(s)$ is reminiscent of the stick-slip motion, but the rearrangement of the atoms leading to an ordered structural transition is rather unexpected. After the transition $T_{1},\left|F_{L}(s)\right|$ increases quasi-linearly with the displacement of the asperity. Once the asperity moves approximately $2 \AA$, or by the height of the $2 \mathrm{D}$ hexagonal unit cell of the $\mathrm{Ni}(111)$ planes, the stick behavior ends and the slip starts with the second structural transition $M_{1}$. The side views of the atoms before and after the transition $M_{1}$ are shown in Figs. 9(c) and 9(d); those corresponding to $x y$ projections are presented in Figs. 9(f) and 9(g). Two asperity layers both were commensurate with the $\mathrm{Cu}(110)$ plane [i.e., one produced by the transition $T_{1}$ as explained above and the $\mathrm{Ni}(110)$ layer below it] merge into a single (111) layer of $\mathrm{Ni}$. The transition starts in front of the Ni asperity and ends at its back, and $\left|F_{L}(s)\right|$ decreases abruptly. Note that upon this latter structural transition, one layer of asperity disappears and hence the slip ends with a wear. During the stick stage from $T_{1}$ until the onset of $M_{1}$ the energy of motion is converted into the potential energy and is stored as the strain energy of $\Delta E_{s} \sim 2.8 \mathrm{eV}$. While part of $\Delta E_{s}$ is kept in the new phase on the Born-Oppenheimer surface as a result of the structural transition labeled by $M_{1}$, the major part, $\Delta E_{d} \sim 2$ $\mathrm{eV}$, is, however, released to generate local nonequilibrium phonons and hence is dissipated in the whole system. After $M_{1}, F_{L}(s)$ increases again linearly with $s$; same events repeat leading to transitions $T_{2}$ and $M_{2}$. At each period, the asperity loses one layer, so its contact area with the $\mathrm{Cu}(110)$ surface further increases.

The incommensurate-commensurate structural transition illustrated in Fig. 9 have interesting features. The transition occurs in a short time interval; starting from one ordered 2D structure it ends up in a different ordered atomic structure. In the course of these transitions, the energy of the system makes a sudden change. These features suggest that each structural transition is associated with a first-order phase transition and corresponds to local minimum in the BornOppenheimer surface. This conclusion is strengthened by the analysis of the structure factor, ${ }^{33} S(\vec{q})$.

In closing this part we comment on the following points: (i) The junction between the asperity and the $\mathrm{Cu}$ surface is growing with sliding. The cross section of the $\operatorname{contact} A$ is in the range of $\sim \pi \lambda_{F}^{2}$, where the electronic states are quantized in the $x y$ plane with a level spacing $\sim 10^{3} \mathrm{~K}$. The number of contact states that dip below the Fermi level, $E_{F}$ depends strongly on $A$ and its shape, and the conduction channels derived therefrom are intermixed owing to the tunneling and scattering. ${ }^{22,34}$ Therefore, the ballistic conductance of the contact, $G(s)$ cannot yield sharp quantized step structure even if the contact would evolve adiabatically with sliding. However, $G(s)$ is expected to show abrupt jumps only due to the discontinuous growth of the junction through the $T$ - and $M$-type phase transitions in the course of sliding. 


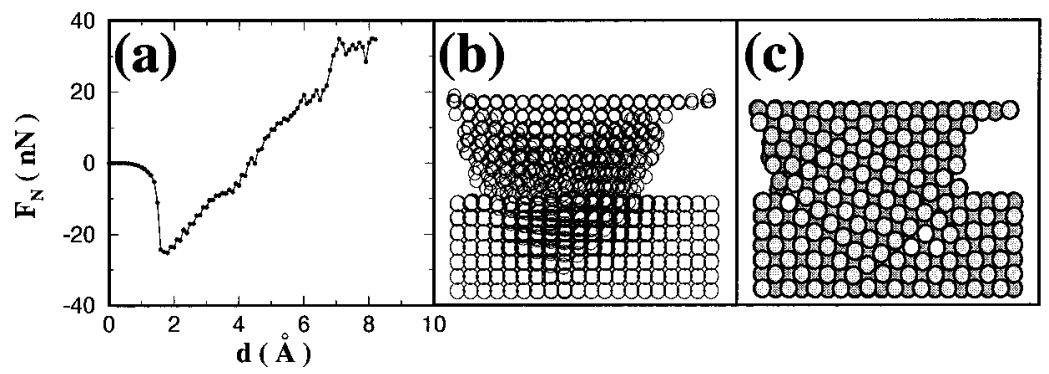

FIG. 10. (a) Variation of the perpendicular force with the displacement of the Ni hemisphere making a contact and then indentation on the $\mathrm{Cu}$ surface. (b) Side views of the atoms during indentation under the repulsive perpendicular force $F_{N}=24 \mathrm{nN}$. (c) Side view of atoms in a cut-plane bisecting the junction corresponding to $F_{N}$ relatively larger than in (b). Note the slip planes.

By measuring $F_{L}(s)$ and $G(s)$ simultaneously in the course of sliding one can provide additional information about the contact. (ii) As the sliding continues, a cluster of atoms grows at the contact behind the asperity. The atoms of this cluster are not usually in disordered state. (iii) The sliding occurs at the interface between $\mathrm{Ni}(111)$ plane and $\mathrm{Ni}(110)$ plane matched to the $\mathrm{Cu}(110)$ plane, since the relative motion between the (111) planes requires relatively lesser energy. (iv) The average friction force $\bar{F}_{L}$ is calculated for $0<s<11 \AA$ to be $-2.06 \mathrm{nN}$. (v) Finally, we note that the usual stick-slip behavior was also obtained in the earlier simulation of the $\mathrm{Si}(111)$ tip sliding on the $\mathrm{Si}(111)$ surface. ${ }^{35}$ Here, the sliding leads to wear and two stick-slip stage in each period are associated with different structural transformations. The mechanism of wear and accumulation of material behind the tip are also results revealed from the present study.

\section{BLUNT TIP}

The blunt tip is constructed from a hemisphere comprising $1580 \mathrm{Ni}$ atoms, which are arranged initially in 14 $\mathrm{Ni}(001)$ planes. The dynamic atoms are in the first nine layers from the bottom; the remaining five $\mathrm{Ni}(001)$ layers at the top are robust. The bottom of the hemisphere is flat and the lowest $\mathrm{Ni}(001)$ layer includes $24 \mathrm{Ni}$ atoms facing the surface of the substrate. The substrate by itself is made from 10 $\mathrm{Cu}(001)$ planes; each plane including $200 \mathrm{Cu}$ atoms, altogether $2000 \mathrm{Cu}$ atoms. The last (bottom) three $\mathrm{Cu}(001)$ layers are robust. The $\mathrm{Ni}(001)$ planes as well as the $\mathrm{Cu}(001)$ planes are parallel to the $x y$ plane. The present case is different from that of the sharp tip, since the $\mathrm{Ni}(001)$ planes are pseudomorphic to the $\mathrm{Cu}(001)$ planes. The lowest part of the tip being flat, the $\mathrm{Ni}$ and $\mathrm{Cu}$ atoms have similar coordination numbers and the hemisphere exerts strong forces on the substrate and can sustain strong forces without significant deformation.

\section{A. Contact and indentation}

The Ni hemisphere (with flat bottom) is lowered by increments of $\Delta z=0.1 \AA$; between two consecutive increments all the dynamic atoms (in the $\mathrm{Ni}$ hemisphere and in the substrate) are relaxed for 500 time steps. When the separation between the lowest layer of the $\mathrm{Ni}$ hemisphere and the $\mathrm{Cu}$ surface is in the range of the cubic lattice parameters $a$ ( $\simeq 3.5 \AA$ ), a significant attractive (adhesion) force is generated; that causes, in particular, the $\mathrm{Cu}$ atoms facing the $\mathrm{Ni}$ hemisphere to jump to contact. It can be understood by the fact that the elastic stiffness constant $C_{11}$ of the $\mathrm{Cu}$ and $\mathrm{Ni}$ crystals are 1.8 and $2.6 \mathrm{nN} / \AA^{2}$. The jump to contact is clearly seen in Fig. 10(a), in which $F_{N}$ attained $\sim 25 \mathrm{nN}$ within a small displacement $d$. Subsequent to the jump to contact $F_{N}$ decreases in the attractive range with increasing $d$. Multiple jump to contacts of the sharp tip in Fig. 2 do not occur in the present situation. While the $\mathrm{Ni}$ atoms forming the contact with the substrate enters gradually to the repulsive range, the $\mathrm{Ni}$ atoms slightly above the contact are still in the attractive range. In the repulsive range, the substrate atoms under the contact is lowered while surface atoms around the contact raised under the attractive interaction with the $\mathrm{Ni}$ atoms. The massive deformation at the close proximity of the contact occurs in the repulsive range, and it involves the substrate atoms under the contact area as seen in Fig. 10(b). In the hemisphere, all the $\mathrm{Ni}$ atoms except those near the center are displaced. The plastic deformations are induced by dislocations in the hemisphere and by slips both in the hemisphere and in the substrate. Fig. 10(c) illustrates the formation of slip planes. We note that $F_{N}(d)$ is piecewise linear in the interval $2<d<7 \AA$. The yielding occurs for $d \sim 7 \AA$; the Ni layers are deformed as a result of compressive strain, and eventually become disordered. Subsequently, the layer structure is recovered, but one layer of the hemisphere disappears and the contact area increases. In the meantime, the $\mathrm{Cu}$ and $\mathrm{Ni}$ atoms start to mix in the interface.

\section{B. Sliding}

The sliding of the blunt Ni tip described in Sec. III on the $\mathrm{Cu}(001)$ surface is investigated under the loading force $F_{N}$ $=9.1 \mathrm{nN}$ and $F_{N}=12.9 \mathrm{nN}$. The lowest atomic plane of the blunt tip, which includes $24 \mathrm{Ni}$ atoms, is initially in contact with the $\mathrm{Cu}(001)$ surface. The interface created by these atoms are rather large and flat. Therefore, the sliding friction is expected to be different from the friction of a sharp tip, treated in Sec. II C.

These two surfaces, i.e., the $\mathrm{Ni}(001)$ and $\mathrm{Cu}(001)$, are pseudomorphic, but their elastic parameters and binding energies are different. In this respect, the relative motion of these two surfaces and resulting friction may involve interesting features. Here the diagonal of the primitive square unit cell is taken parallel to the $x$ direction. In Fig. 11(a) we show the variation of the lateral force under the constant normal loading force, $F_{N}=9.1 \mathrm{nN}$. The variation of the normal reaction force that is calculated in the course of simulations is presented by the inset. It demonstrates that the operation un- 

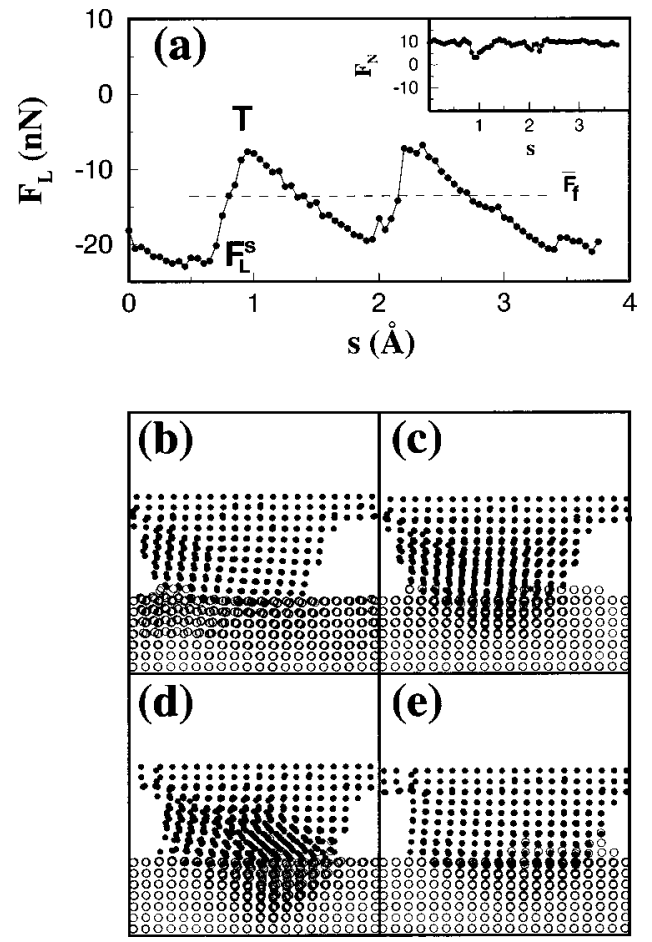

FIG. 11. (a) Lateral force $F_{L}$ vs the lateral displacement $s$ calculated for the Ni hemisphere sliding on the $\mathrm{Cu}(001)$ surface under the loading force, $F_{N}=9.1 \mathrm{nN}$. The inset show that the loading force does not vary significantly. $F_{L}$ is the lateral force before the first slip stage begins. $T$ indicates the point where the first slip ends and the second stick stage starts. (b) and (c) show the side view of atomic structure in the stick stages before and after a slip taking place under the loading force, $F_{N}=9.1 \mathrm{nN}$. $\mathrm{Ni}$ and $\mathrm{Cu}$ atoms are shown by filled and empty circles. (d) and (f) are stick stages before and after a slip taking place under a larger loading force, $F_{N}$ $=12.9 \mathrm{nN}$. $\mathrm{Ni}$ and $\mathrm{Cu}$ atoms are shown by filled and empty circles.

der the constant loading force mode is well simulated. Figs. 11(b)-(e) show the atomic structure before and after the slip for different loading forces. We note that in Fig. 11(a) the ratio of the static friction force $F_{L}^{S}$ and average kinetic friction force, $\bar{F}_{f}$, i.e., $F_{L}^{S} / \bar{F}_{f} \sim 2$. In the low loading (i.e., $F_{N}$ $=9.1 \mathrm{nN}$ ) case the $\mathrm{Ni}$ surface is initially stuck on the $\mathrm{Cu}$ surface; whole $\mathrm{Ni}$ hemisphere and the $\mathrm{Cu}$ surface are sheared and line dislocations are generated on the substrate surface below the contact interface until the slip sets in. During slip the atomic structure at the contact becomes disordered, some $\mathrm{Cu}$ and $\mathrm{Ni}$ atoms are exchanged and the hemisphere is displaced along the [110] direction. Eventually the regular structure at the contact is recovered upon the jump of the $\mathrm{Ni}$ atoms at the interface to their new positions. These events take place in a short interval, and the lateral force $F_{L}$ decreases by $\sim 15 \mathrm{nN}$. As the sliding continues following the slip the hemisphere and the substrate at the junction are sheared again in the new stick stage started at $T$. While in the front of the hemisphere the junction becomes disordered and more atoms are exchanged, in the back the slips occur on the $\mathrm{Ni}(111)$ planes. At the end, the quasilinear increase of the lateral force $F_{L}(s)$ stops with the start of the slip stage; it decreases suddenly while the $\mathrm{Ni}$ atoms at the contact jump to the next position by moving along the [1ㅣㅣ direction. For large $F_{N}$, the generation of dislocation and slip planes in the hemisphere become pronounced in the sliding motion; this way the stick-slip motion becomes less regular.

\section{CONCLUSIONS}

We presented the detailed analysis of contact formation, indentation, subsequent pulling and dry sliding friction of the $\mathrm{Ni}$ tip on the relatively soft $\mathrm{Cu}$ surface. We investigated the effect of tip geometry (sharp or blunt), crystal structure of the tip and sample, and discovered the following important features: (i) Upon the onset of the contact of a sharp tip the multiple jump to contact events can occur in the attractive regime of the normal force. The Ni layers (or atoms) near the apex engage in the jump to contact phenomena even though the elastic stiffness constants of $\mathrm{Ni}$ are larger than those of $\mathrm{Cu}$. Already with the onset of the contact, atoms from the lower planes of the asperity become matched to the substrate lattice structure. The situation is reversed if the tip is blunt with large area of contact. In this case, mainly the $\mathrm{Cu}$ atoms on the surface jump to contact in a single event. (ii) Indentation proceeds in the repulsive force range. For a sharp tip the perpendicular force displays quasilinear variations followed by a sudden decrease caused by disappearance of a layer, or other structural changes. The junction grows discontinuously and, in general, suddenly by the implementation of the new atoms to the interface. However, the number of atoms that are brought to contact is different for each growth event. It is interesting to explore whether the growth of the contact in terms of these new atoms is correlated with the electronic contact states dipping into the Fermi level. Such effects were explored earlier by 2D metals. ${ }^{1}$ In the indentation of the flat tip the sudden force variations (or yields) are not so frequent and occurs mainly through the slips on the atomic planes. (iii) The atom exchange, in which the substrate and the asperity atoms change their positions occurs in the repulsive force range and increases with increasing deformation. (iv) Pulling off from an indentation occurs in the attractive (tensile) range of the perpendicular force. The connective neck first forms through interesting atomic rearrangements and then is broken leaving a $\mathrm{Ni}$ pyramidal asperity on the $\mathrm{Cu}$ surface, that is lattice matched to the substrate. (v) During the pulling off, the tensile force exhibits abrupt changes. However, each of these changes are not associated with the creation of a new layer. Sometimes, a single atom implemented between two adjacent layers induces additional structures in force variation. (vi) In the dry sliding of a sharp $\mathrm{Ni}(111)$ tip (or asperity) on the $\mathrm{Cu}$ surface under constant loading force the lateral force exhibits quasi periodic variation; each period consists of two different stick-slip process involving structural phase transitions. While one layer of asperity changes and matches the substrate lattice in the first slip, two asperity layers merge into a one through structural transition during the second slip. This leads to wear. While the relative motion occurs between the regular plane and the substrate matched plane of the asperity, the strain energy is stored at the interface. (vii) The sliding of a flat $\mathrm{Ni}(001)$ surface on the $\mathrm{Cu}(001)$ surface depends on the loading force. In the low loading case, the lateral force displays quasilinear variation in the stick stage that is followed by the sudden decrease in the slip stage. For large loading, the stick-slip motion is less regular. ${ }^{36}$ 
${ }^{1}$ S. Ciraci and I. P. Batra, Phys. Rev. B 33, 4294 (1986); I. P. Bayra, S. Ciraci, G. P. Srivastava, J. S. Nelson, and C. Y. Fong, 34, 8246 (1986).

${ }^{2}$ J. K. Gimzewski and R. Möller, Phys. Rev. B 36, 1284 (1987).

${ }^{3}$ N. Agrait, J. G. Rodrigo, and S. Vieria, Phys. Rev. B 47, 12345 (1993).

${ }^{4}$ N. Agrait, G. Rubio, and S. Vieria, Phys. Rev. Lett. 74, 3995 (1995); G. Rubio, N. Agrait, and S. Vieria, ibid. 76, 2302 (1996).

${ }^{5}$ A. Stalder and U. Dürig, Appl. Phys. Lett. 68, 637 (1996).

${ }^{6}$ S. Ciraci and E. Tekman, Phys. Rev. B 40, 11969 (1989).

${ }^{7}$ T. N. Todorov and A. P. Sutton, Phys. Rev. Lett. 70, 2138 (1993); A. M. Bratkovsky, A. P. Sutton and T. N. Todorov, Phys. Rev. B 52, 5036 (1995)

${ }^{8}$ K. L. Johnson, Contact Mechanics (Cambridge University Press, Cambridge, 1986).

${ }^{9}$ S. Ciraci, in Tip-Surface Interactions in Scanning Tunneling Microscopy and Related Methods, Vol. 183 of NATO Advanced Study Institute, Series E: Applied Science, edited by R. J. Behm, N. Garcia, and H. Rohrer (Kluwer Academic, Dordrecht, 1990) p. 113; E. Tekman and S. Ciraci, Phys. Rev. B 40, 10286 (1989); E. Tekman and S. Ciraci, Phys. Scr. 38, 486 (1988).

${ }^{10}$ C. M. Mate, G. M. McClelland, R. Erlandsson, and S. Chiang, Phys. Rev. Lett. 59, 1942 (1987).

${ }^{11}$ For a current review see, for example, Physics of Sliding Friction, Vol. 311 of NATO Advanced Study Institute, Series E: Applied Science, edited by B. N. J. Persson and E. Tosatti (Kluwer Academic, Dordrecht, 1996; Micro/Nanotribology and its Applications, edited by B. Bhushan (Kluwer Academic, Dordrecht, 1997).

${ }^{12}$ G. J. Germann, S. R. Cohen, G. Neubauer, G. M. McClelland, and H. Seki, J. Appl. Phys. 73, 1 (1993); S. Fujisawa, E. Kishi, Y. Sugawara, and S. Morita, Phys. Rev. B 51, 7849 (1995).

${ }^{13}$ M. Hirano and K. Shinjo, Phys. Rev. B 41, 11837 (1990).

${ }^{14}$ M. Hirano, K. Shinjo, R. Kaneko, and Y. Murata, Phys. Rev. Lett. 67, 2642 (1991).

${ }^{15}$ K. Shinjo and M. Hirano, Surf. Sci. 283, 473 (1993).

${ }^{16}$ S. R. Cohen, G. Neubauer, and G. M. McClelland, J. Vac. Sci. Technol. A 8, 3449 (1990).
${ }^{17}$ A. P. Sutton and J. B. Pethica, J. Phys.: Condens. Matter 2, 5317 (1990); J. A. Nieminen, A. P. Sutton, and J. B. Pethica, Acta Metall. Mater. 40, 2503 (1992).

${ }^{18}$ S. Ciraci, E. Tekman, A. Baratoff, and I. P. Batra, Phys. Rev. B 46, 10411 (1992).

${ }^{19}$ M. R. Sørensen, K. W. Jacobsen, and P. Stoltze, Phys. Rev. B 53, 2101 (1996); M. R. Sørensen, K. W. Jacobsen, and H. Jónsson, Phys. Rev. Lett. 77, 5067 (1996).

${ }^{20}$ S. Ciraci and I. P. Batra, Phys. Rev. B 36, 6194 (1987).

${ }^{21}$ S. Ciraci, A. Baratoff, and I. P. Batra, Phys. Rev. B 42, 7618 (1990).

${ }^{22}$ E. Tekman and S. Ciraci, Phys. Rev. B 43, 7145 (1991).

${ }^{23}$ E. Tekman and S. Ciraci, Phys. Rev. B 42, 1860 (1990).

${ }^{24}$ F. F. Abraham, I. P. Batra, and S. Ciraci, Phys. Rev. Lett. 60, 1314 (1988).

${ }^{25}$ U. Landman, W. D. Luedke, N. A. Burnham, and R. J. Colton, Science 248, 454 (1990).

${ }^{26}$ M. Brandbyge, J. Schiøtz, M. R. Sørensen, P. Stoltze, K. W. Jacobsen, and J. K. Nørskov, Phys. Rev. B 52, 8499 (1995).

${ }^{27}$ For a critical review see also A. P. Sutton, Curr. Opin. Solid State Mater. Sci. 1, 827 (1996).

${ }^{28}$ M. S. Daw and M. I. Baskes, Phys. Rev. B 29, 6443 (1984); S. M. Foiles, M. I. Baskes, and M. S. Daw, ibid. 33, 7983 (1986); M. S. Daw, ibid. 39, 7441 (1989). We used the embedded atom model potentials for $\mathrm{Cu}$ and $\mathrm{Ni}$ developed in the above works.

${ }^{29}$ S. P. Jarvis, H. Yamada, S. I. Yamamoto, H. Tokumoto, and J. B. Pethica, Nature (London) 384, 247 (1996).

${ }^{30}$ J. K. Gimzewski, R. Möller, D. W. Pohl, and R. R. Schlitter, Surf. Sci. 189, 15 (1987).

${ }^{31}$ H. Mehrez, M.S. thesis, Bilkent University, 1996.

${ }^{32}$ H. Mehrez and S. Ciraci, Phys. Rev. B 56, 12632 (1977); J. Phys. 9, 10843 (1977).

${ }^{33}$ A. Buldum and S. Ciraci, Phys. Rev. B 55, 12892 (1977).

${ }^{34}$ H. Mehrez, S. Ciraci, A. Buldum, and I. P. Batra, Phys. Rev. B 55, R1981 (1997).

${ }^{35}$ U. Landman, W. D. Luedke, and A. Nitzan, Surf. Sci. 210, L177 (1989).

${ }^{36}$ A. Buldum and S. Ciraci, Phys. Rev. B 55, 2606 (1997). 